\title{
Volume measurement of cryogenic deuterium pellets by Bayesian analysis of single shadowgraphy images
}

\author{
T. Szepesi ${ }^{1}$, S. Kálvin ${ }^{1}$, G. Kocsis ${ }^{1}$, P.T. Lang ${ }^{2}$, C. Wittmann ${ }^{2}$ and ASDEX Upgrade Team ${ }^{2}$ \\ ${ }^{1}$ KFKI Research Institute for Particle and Nuclear Physics, EURATOM Association, \\ P.O. box 49, H-1525 Budapest-114, HUNGARY \\ ${ }^{2}$ Max-Planck-Institut für Plasmaphysik, EURATOM Association, \\ Boltzmannstr. 2, 85748 Garching, GERMANY
}

\begin{abstract}
In situ commissioning of the Blower-gun injector for launching cryogenic deuterium pellets at ASDEX Upgrade tokamak was performed. This injector is designed for high repetitive launch of small pellets for ELM pacing experiments. During the investigation the final injection geometry was simulated with pellets passing to the torus through a $5.5 \mathrm{~m}$ long guiding tube. For investigation of pellet quality at launch and after tube passage laser flash camera shadowgraphy diagnostic units before and after the tube were installed. As indicator of pellet quality we adopted the pellet mass represented by the volume of the main remaining pellet fragment. Since only $2 D$ shadow images were obtained, a reconstruction of the full $3 D$ pellet body had to be performed. For this the image was first converted into a 1-bit version prescribing an exact $2 D$ contour. From this contour the expected value of the volume was calculated by Bayesian analysis taking into account the likely cylindrical shape of the pellet. Under appropriate injection conditions sound pellets with more than half of their nominal mass are detected after acceleration; the passage causes in average an additional loss of about $40 \%$ to the launched mass. Analysing pellets arriving at tube exit allowed for deriving the injector's optimized operational conditions. For these more than $90 \%$ of the pellets were arriving with sound quality when operating in the frequency range $5-50 \mathrm{~Hz}$.
\end{abstract}

\section{Introduction}

During the last decades cryogenic hydrogen isotope pellets have been successfully applied for plasma particle replenishment of magnetic confinement fusion experiments. Fuelling pellets injected during high confinement mode (H-mode) plasma phases ${ }^{1}$, have been found to act also as triggers ${ }^{23}{ }^{4}$ for MHD instabilities such as type-I Edge Localised Modes (ELMs), which also can be triggered promptly. ELMs, also appearing spontaneously in most H-mode regimes ${ }^{5}$, cause a sudden loss of particles and energy from the plasma edge into the scrape-off layer (SOL), resulting in a considerable peak power load on first wall components. This transient power load scales with machine size, and will reach unacceptably high levels for large tokamaks like ITER ${ }^{6}$. However, ELMs also remove impurities from the plasma, making the type-I ELMy H-mode a robust, stable operating scenario ${ }^{7}$. The stabilising effect of type-I ELMs makes the ELMy H-mode a suitable candidate for the standard operating scenario of fusion power plants. Therefore it is required to develop methods for mitigation of unwanted ELM effects. One of these methods is ELM frequency control by continuous pellet injection (pacemaking), demonstrated at ASDEX Upgrade tokamak ${ }^{8}$. It was found in ELM pacemaking 
experiments that - just like for natural ELMs - the energy loss caused by a triggered ELM $\left(\Delta W_{\text {ELM }}\right)$ in steady state is inversely proportional to the ELM frequency $\left(f_{\text {ELM }}\right)$, thus increasing $f_{\text {ELM }}$ beyond the natural ELM frequency $\left(f_{\text {ELM }}^{0}\right.$ ) results in a decrease of $\Delta W_{\text {ELM }}$. Full control over the ELM frequency can be achieved by increasing the pellet injection frequency to a sufficiently high value compared to the natural ELM frequency: $f_{\text {pel }} \geq 1.5 f_{\mathrm{ELM}}^{0}{ }^{8}$. For a reasonable ELM mitigation, however, quite larger values for this frequency enhancement ratio must be achieved, thought to range around 10-40. Accordingly high pellet injection frequencies, on the other hand, cause a high fuelling burden, as presently the achievable smallest solid hydrogen pellet size is in the mm range (pellet particle content several $10^{19}$ atoms). Within present tokamak sizes this can alter already significantly the total plasma particle inventory. Although this burden eases with growing machine size, pellet pacing experiments in present machines can only be conducted if the additional fuelling does not hamper target plasmas. Therefore, a pellet launcher ("Blower-gun”) optimised for ELM triggering was developed at ASDEX Upgrade tokamak (AUG), featuring low field side (LFS) injection with small sized pellets ${ }^{9}$. This Blower-gun was commissioned in the C2006 campaign at AUG, however the pellet delivery efficiency into the plasma observed was much lower than during the laboratory test phase, implying that the small-size pellets are broken on their track into the plasma. Therefore, a shadowgraphy camera diagnostic system was installed to inspect pellets during their flight, and to identify whether pellets are broken during acceleration or in the guiding tube. The results of these measurements will be presented in this paper.

Another well-known method to determine the volume of fast projectiles is the microwave cavity diagnostic, where the change in the cavity's resonant frequency due to the presence of dielectric material (pellet) is inspected ${ }^{10}$. However, the technical effort to realize a working microwave cavity diagnostic at ASDEX Upgrade would have been too high compared to the limitations of this diagnostic, namely: the microwave cavity cannot distinguish between a single normal size pellet and several pellet fragments (with a total mass equal to a full size pellet) arriving to the cavity in the same time. However, the ability to distinguish these cases is one of the key issues in the present study, as explained in detail in section 3.

Getting back to the shadowgraphy diagnostic, the fundamental problem to solve is to derive the real volume of a 3D object from its 2D shadow images. In principle a precise volume determination could be obtained (at least for convex objects) from several shadow images, but for this plenty of different views at the same position are required, and the directions of the views depend on the object's shape. The technical effort to realize this kind of measurement is really high even in the case when the shape of the object and also its orientation are known. In case of pellet injection neither of these properties is exactly known, therefore only one view was used in a shadowgraphy diagnostic unit for the Blower-gun.

In this setup the shape of the pellet shadow can vary from pellet to pellet because of two reasons: first, the pellets can freely rotate in the guiding tube and can arrive to the shadowgraphy location with different sides visible (orientation); second, the pellet shape and size itself may vary because of fragmentation and/or evaporation etc (size reduction). Therefore a Bayesian analysis, considering shadow shape changes due to both orientation and size reduction on a probabilistic basis was applied to estimate the pellet volume from its single 2D shadowgraphy image. Results are presented in this paper.

This paper is organised as follows: section 2 describes the experimental setup for the testing of the Blower-gun; the evaluation of the shadowgraphy images, as well as the Bayesian volume reconstruction analysis together with its testing are presented in section 3; results are detailed in section 4, and finally section 5 presents the conclusions. 


\section{Apparatus}

The Blower-gun delivers cylindrical pellets with a diameter of $2 \mathrm{~mm}$ and a length in the range of $0.5-2 \mathrm{~mm}$ (in $0.5 \mathrm{~mm}$ steps). In the present setup the cryostat provides a $124 \mathrm{~mm}$ long rod of deuterium ice, pellets are cut from this ice rod, allowing for a pellet string containing ca. 110 pellets of $1 \mathrm{~mm}$ length, while some ice is lost due to technical reasons. The acceleration mechanism is the blower-gun principle: pellets are accelerated by the viscous drag force between the pellet surface and a short, high pressure gas pulse (propellant gas) travelling with about sound speed in an acceleration barrel with a diameter considerably larger than the pellet size. The gas pulse is released from the reservoir by a fast gas valve. This method does not provide a well defined pellet speed: e.g. with the standard settings $\left(D_{2}\right.$ propellant gas at $p_{\text {prop }}=3.5 \mathrm{bar}$, pulse length: $\tau_{\text {valve }}=2 \mathrm{~ms}$ ) pellets reach a velocity in the range of $150-250 \mathrm{~m} / \mathrm{s}$. Available repetition rates of the injector are: $f_{\mathrm{pel}}=5-143 \mathrm{~Hz}^{9}$.

\subsection{Experimental setup}

The Blower-gun was installed for an in situ off-line test in a geometry conservatively modelling the AUG injection geometry. Two shadowgraphy diagnostic units were used, one located directly after the Blower-gun (to study the effect of the acceleration) and another behind the guiding tube, directly in the part connecting to the torus (to study the effect of the guiding tube). A guiding tube of $5.5 \mathrm{~m}$ length and $6 \mathrm{~mm}$ inner diameter, as required for pellet transfer to the torus, was installed. Although studying the effect of the guiding tube setup on the delivery efficiency was not part of this paper, valuable information can be gained on the topic from previous investigations at ASDEX Upgrade and $\mathrm{JET}^{11}{ }^{12}$. According to these studies a larger inner diameter guiding tube leads to efficiency loss, just like the two small curvature radii $(\mathrm{R}<1 \mathrm{~m})$ in the present setup. Therefore the number of sound pellets is expected to be notably higher before the guiding tube. In this way the present setup provides a highly conservative estimate for on-line measurements where the guiding tube follows an almost straight path to the torus. The off-line test setup can be seen in Figure 1.

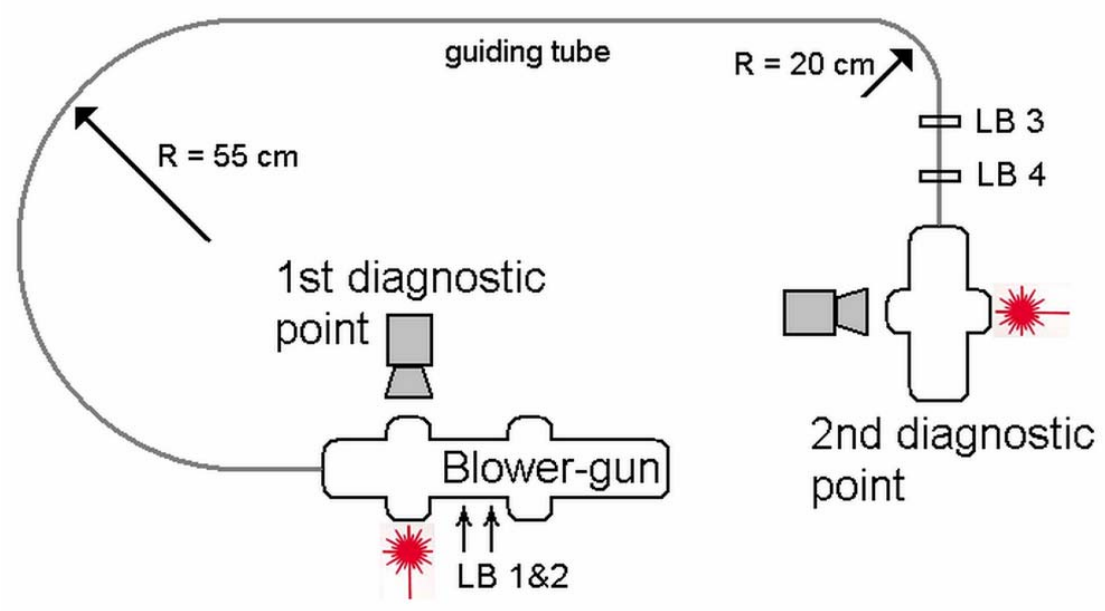

Figure 1.: Experimental setup - Blower-gun, guiding tube, two shadowgraphy diagnostics. Total guiding tube length $5.5 \mathrm{~m}$. 
Every shadowgraphy diagnostic unit consists of a PCO Pixelfly camera, a pulsed IR laser (acting as a flash) and a free-flight region in the guiding tube to allow clear observation of the pellet. The used image resolution is $320 \times 120$ pixels (horizontal binning: 2, vertical: 4) with a 12-bit dynamic range. This resolution allows for a frame rate up to $170 \mathrm{~Hz}$, well beyond the Blower-gun's repetition rate. However, this high frame rate could only be achieved for the $2^{\text {nd }}$ shadowgraphy unit; the camera in the $1^{\text {st }}$ unit had a maximum frame rate of $50 \mathrm{~Hz}$, meaning that a correction factor had to be used when determining the injector's efficiency in scenarios with pellet injection frequencies higher than $50 \mathrm{~Hz}$. The correction factor was taken as the ratio between the given and accepted triggers. Before the evaluation all images were interpolated to $320 \times 240$ pixels to restore 1:1 aspect ratio.

In the measurement the pellet travels in-between the laser flash and the camera, therefore its shadow is recorded as an image - this method allows for a very high contrast observation despite the pellet's $\sim 200 \mathrm{~m} / \mathrm{s}$ velocity, as the laser pulse length is $1 \mu \mathrm{s}$. Because of the considerable scatter in the pellet velocity $\left(v_{\mathrm{pel}}\right)$, the shadowgraphy cameras and the lasers must have a triggering system that can compensate for the varying pellet speed for a wide range of velocities. This is realised by a pair of light barriers for each shadowgraphy unit (LB 1\&2 for unit 1 and LB 3\&4 for unit 2): the first LB (1 or 3) starts a counter counting up from 0 with frequency $f_{1}$, while the second LB (2 or 4) stops the counter and starts a count-down from the current value of the counter $(N)$ with frequency $f_{2}$ (the two shadowgraphy units have separate counters). A trigger signal is produced when the counter reaches zero again. If $d$ is the distance between LB 1(3) and LB 2(4), and $x$ is the distance from LB 2(4) to the shadowgraphy location, and if equation

$$
\Delta t_{1} f_{1}=N=\Delta t_{2} f_{2} \Leftrightarrow \frac{d}{v_{p e l}} f_{1}=N=\frac{x}{v_{p e l}} f_{2}
$$

is satisfied, the trigger is provided exactly when the pellet reaches the shadowgraphy location (see Figure 2). We can assume $v_{\text {pel }}$ to be constant as the pellet travels only about $0.5 \mathrm{~m}$ in the diagnostic section. By recording $N$, which is possible at the second shadowgraphy location, the pellet velocity can also be determined from $x$ and $f_{2}$. This way the shadowgraphy triggering system is suitable to produce triggers for the shadowgraphy lasers and cameras and to study the pellet velocity distribution at the same time.

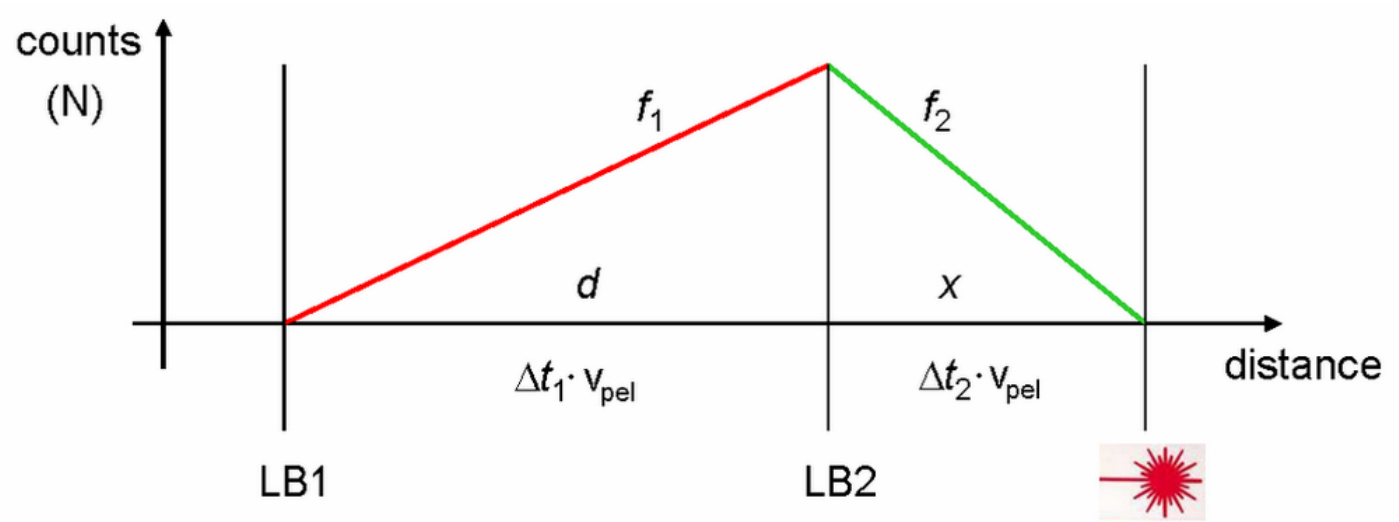

Figure 2.: Principle of the shadowgraphy triggering system. 


\subsection{Calibration of the images}

To convert the dimensions of the pellet shadow on the image into physical dimensions, a special calibration target was produced: a concentric circular pattern was engraved into a plexiglass cylinder, the separation between the circles being $0.5 \mathrm{~mm}$. This object was placed into the measurement positions when the system was vented. The circular pattern allows for the determination of the conversion coefficient between image pixels and real length units as well as the dependence of this coefficient on the image coordinates (distortion) by fitting regular circles on the image. In case the circles fit well to the image, the distortion in the $x$ and $y$ directions is isotropic. If the distance between the fitted circles (in pixels) is also uniform, the imaging has no detectable distortion and a single conversion coefficient can be used. The results are shown on Figure 3.
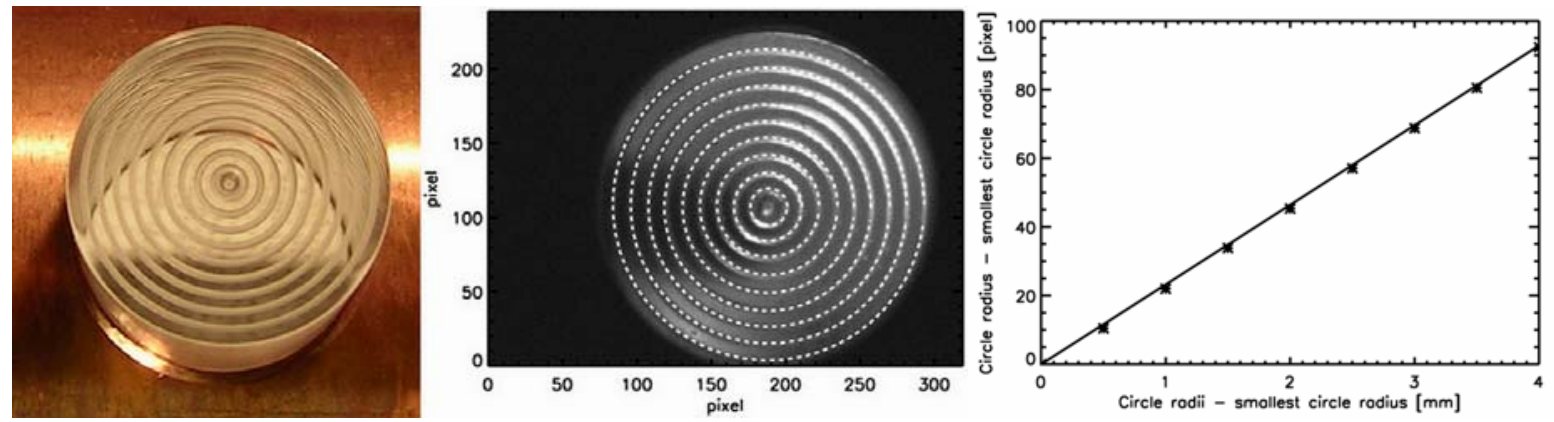

Figure 3.: Spatial calibration of shadowgraphy diagnostic. Calibration target (left), view of $2^{\text {nd }}$ diagnostic position (middle) overlaid are calibration circles (dashed lines). Circle radius in pixels versus circle radius in $\mathbf{m m}$ (right).

The calibration procedure has shown that the above assumptions are correct: the drawn circles fit well to the image, and their radius increases linearly in pixel number, meaning that distortion is negligible, and a single conversion coefficient between pixels and real units [mm] can be used. This coefficient varies each time the optics are removed, therefore a new calibration is necessary in these cases. The typical value of the conversion factor is 23 pixels/mm, set by the resolution (320x240 pixels) of the image and the magnification of the camera objective.

\section{Pellet volume reconstruction}

Precise knowledge of the pellet volume and hence mass is inevitable for the thorough analysis of the Blower-gun. Also, to be able to compare experiments and pellet ablation modelling, one must know the mass of the launched pellets (simulations require pellet mass as an input parameter). One major aim of pellet simulations is to give good estimates of the pellet penetration depth.

In experiments, penetration measurements are usually based on devices detecting the light emitted by the pellet cloud - photodiodes or cameras. Therefore, when aiming at constructing an algorithm that reconstructs the pellet mass, one also has to take into account the factors having the strongest effect on the light emitted by the pellet. In more detail, the originally intact, cylindrical pellets fired by the Blower-gun are usually fragmented by acceleration and/or during their flight in the guiding tube. Even the whole pellet may be broken into several parts. The most likely case - as observed in preliminary manual analysis of pellet 
shadowgraphy images - is that the pellet is broken into one large and several small fragments. Concerning terminology, one may call each of these fragments a 'pellet' too; but to avoid confusion later, a 'pellet' in this paper refers to a single pellet event (i.e. a fired pellet). In case it is fragmented, 'pellet' refers to the largest piece while all other fragments are disregarded (even if there are only two equal size fragments, only one of them is considered). The point behind this convention comes from penetration studies: the small fragments have shallow penetration and they are barely visible in the plasma: the light produced by the ablating pellet (fragment) is approximately proportional to the ablation rate of the pellet $(\dot{N})$, which according to most pellet ablation models, e.g. the Neutral Gas Shielding (NGS) model ${ }^{13}$ scales with pellet radius: $\dot{N} \sim r_{\text {pel }}^{4 / 3}$. Therefore the visual observation of the penetration depth (into the hot confined plasma) is dominated by the largest fragment, and the small fragments are negligible. Hence, even if several fragments (distinct shadows) of the same pellet are visible on a shadowgraphy image, only the largest fragment will be called 'pellet', and the volume reconstruction algorithm, too, will only be applied for the largest piece on the image.

\subsection{Image evaluation and shadow parameters}

To determine the pellet shadow characteristics, the shadow has to be identified on the image; in case the pellet is broken, there are several distinct shadows to be identified. First, a region-of-interest (ROI) is selected to remove structural elements from the image. Then the image is converted into a 1-bit (black-and-white) version by applying a preset threshold separating black and white corresponding to the shadow and the background, respectively. The threshold is chosen manually (for every shot) in a way not to crop the shadow too much, while avoiding the production of 'fake' shadows due to the non-uniform brightness of the background. Finally contours for all contiguous black patches on the image are determined, supposing that all distinct patches correspond to separate fragments of a single pellet. The number of fragments for each image is also determined. Figure 4 shows an example where the shadow detection algorithm has identified two (a large and a small) distinct shadows.
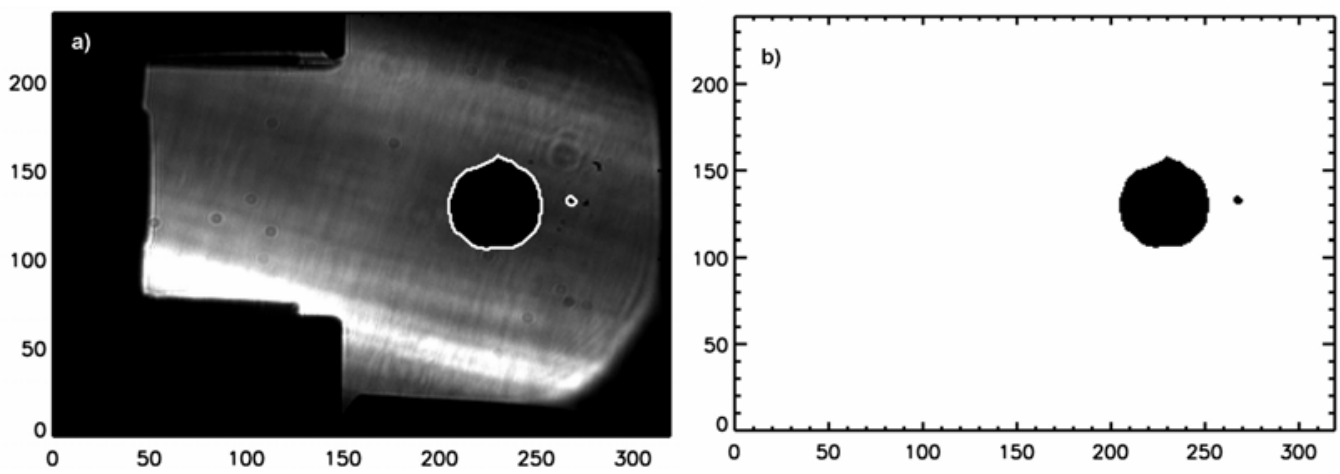

Figure 4.: Raw shadowgraphy image (left) and 1-bit version (right) with two detected pellet fragments - a large and a small one (plot dimensions in pixels). Detected pellet fragment contours are overlaid on the original image.

The shadows identified on the image must now be parameterised. These parameters, the key information for the volume reconstruction, are input variables to the Bayesian algorithm (described in section 3.2). As the pellet can rotate inside the guiding tube, its shadows shape also changes; to characterise the pellet shadow the following three parameters were introduced: shadow area $(A)$, largest dimension $(D)$ and smallest dimension $(L)$. The 
definition of $D$ and $L$ is as follows: the largest and the smallest distance between two parallel lines touching the pellet contour, when the shadow is rotated $180^{\circ}$ in between the lines. These shadow parameters for ideal cylindrical pellets with parameters $r=h=1 \mathrm{~mm}$, where $r$ and $h$ stand for the radius and height of the cylinder, are presented on Figure 5.
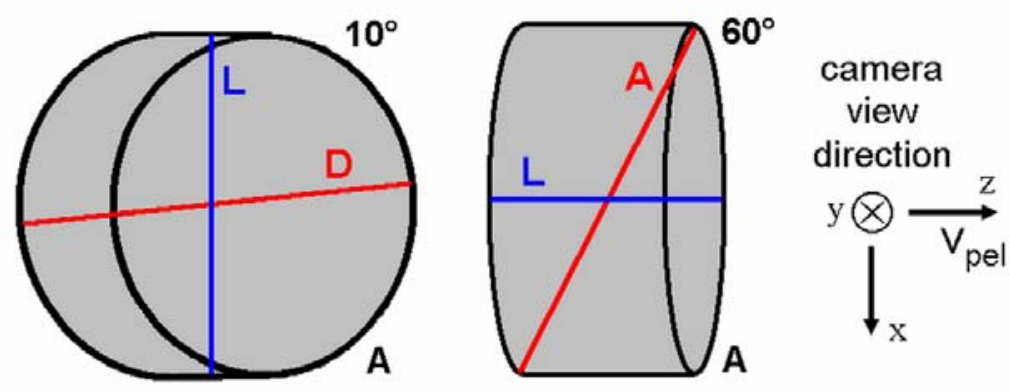

Figure 5.: Pellet shadow parameters for an ideal cylindrical pellet: area $(A)$, largest dimension $(D)$, smallest dimension $(L)$. Pellet rotated by $10^{\circ}$ and $60^{\circ}$ to the camera view direction (y).

The following local coordinate system was used for a shadowgraphy unit: $z$ denotes the pellet flight direction and $y$ stands for the camera view direction (perpendicular to $z$ ); adding an $x$ direction appropriately, a right-handed coordinate system is formed (Figure 5). For a fixed cylindrical pellet shape and given size, the 2D image of the pellet shadow is only determined by the angle $\alpha$ between the camera sight direction $(y)$ and the pellet's orientation (the direction of the surface vector of the pellet's circular base). Of course, the pellet's basevector can point to any direction; however, the shape of the shadow only depends on $\alpha$, as the rotation about the $y$ axis only rotates the shadow, but does not modify its shape. Therefore, $D$, $L$ and $A$ are only depending on $\alpha$. The values for these variables can easily be determined for the extreme values of $\alpha$ in the ideal cylindrical case:

\begin{tabular}{|c|c|c|c|c|}
\hline$\alpha$ & Shadow shape & $L$ & $D$ & $A$ \\
\hline $0^{\circ}$ & circle & $2 r$ & $2 r$ & $r^{2} \pi$ \\
\hline $90^{\circ}$ & rectangle & $\min \{2 r, h\}$ & $\sqrt{4 r^{2}+h^{2}}$ & $2 r \cdot h$ \\
\hline
\end{tabular}

Table 1.: Values of $D, L$ and $A$ for ideal cylindrical pellets (shadow radius $r$ and height $h$ ) at extreme $\alpha$

Two of these parameters for cylindrical pellets can be calculated analytically:

$$
\begin{gathered}
A(r, h, \alpha)=2 r \cdot h \sin (\alpha)+r \cdot r \cos (\alpha) \cdot \pi \\
L(r, h, \alpha)=\min \{h \sin (\alpha)+2 r \cos (\alpha), 2 r\}
\end{gathered}
$$

but the third one (D) only numerically. If we choose $r=h=1 \mathrm{~mm}$, as in the experiment (ideal case), we find that for a considerable range of $\alpha$ these quantities show only a slight variation (see Figure 6). If these plateaus would have a considerable overlap, the parameters would not be useful for reconstructing the pellet volume. Ideally the shadow parameters are continuous functions of the angle $\alpha$; however in the experiment these parameters are step-functions 
because of the finite resolution of the shadowgraphy image (see section 2.2). This effect introduces an additional error source to the volume reconstruction (details in section 3.2).
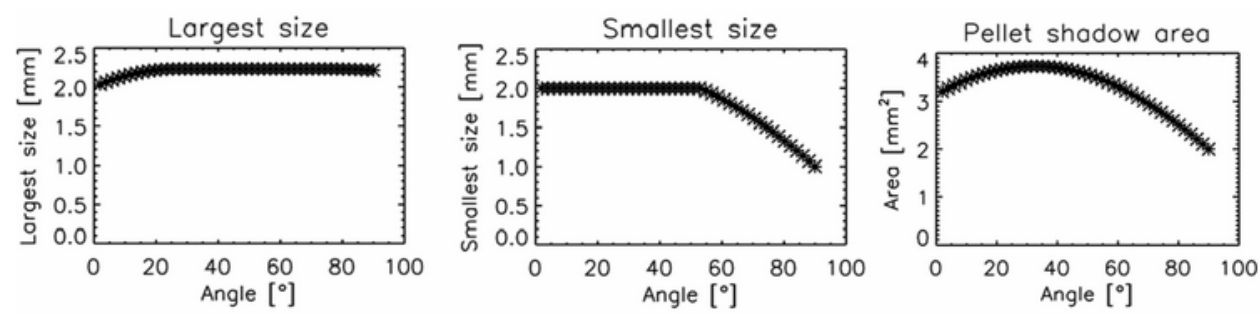

Figure 6.: Pellet shadow parameters for an ideal cylindrical pellet $(r=h=1 \mathrm{~mm})$ as a function of $\alpha$

\subsection{Estimating the pellet volume}

The main problem with the shadowgraphy diagnostic is that from a single 2-dimensional shadow image one cannot reconstruct the volume of a 3-dimensional object, i.e. the pictures are not suitable to determine the pellet mass, they can only be used to tell the number of pieces if the pellet is disintegrated, and to give a very rough estimate of the size of these fragments. However, if we have a reasonable assumption of the shape of the 3D object, we can give a better estimate. For this, it was assumed that the pellet visible on the image preserves its cylindrical shape throughout its flight in the guiding tube, only its two parameters, radius $(R)$ and height $(H)$ are allowed to change. Consequently the shadow parameters $D, L$ and $A$ are then complicated functions of $R, H$ and $\alpha$, even with identical output values for several combinations of $R, H$ and $\alpha$. As a result, it is impossible to determine the exact value of $r, h$ (and $\alpha$ ) based on $d, l$ and $a$, where the capital letters stand for the variables, and lowercase letters denote calculated and measured values. Instead, a Bayesian method was applied to determine the probability distribution function (PDF) of $r$ and $h$.

The basis of the Bayesian analysis is the Bayes' theorem ${ }^{14}$, which can be used to invert the problem: for a given pellet parameter combination $(r, h, \alpha)$ we can determine the likelihood of the shadow parameters $(d, l, a)$; to do it the other way around i.e. to find the posterior PDF of $(r, h, \alpha)$ from measured $(d, l, a)$ values we apply the Bayes' theorem:

$$
P(R, H, \alpha \mid d, l, a)=\frac{P(d, l, a \mid R, H, \alpha) \cdot P(R, H, \alpha)}{\iiint_{R, H, \alpha} P(d, l, a \mid R, H, \alpha) \cdot P(R, H, \alpha)}
$$

The term $P(R, H, \alpha)$ in the equation's right-hand side (RHS) is called the prior - our knowledge about the result before the measurement. With this term one can add expectations to the evaluation, e.g. we expect pellets with similar radius and height, but we do not expect e.g. $H<<R$. In the evaluation, we used a prior $P(R, H, \alpha)=P(R, H) \cdot P(\alpha)=1 \cdot \cos (\alpha)$, i.e. no preference was set for $R$ and $H$, they can vary freely in the given range:

$$
\begin{aligned}
0.1 \mathrm{~mm} & \leq R \leq 1.1 \mathrm{~mm} \\
0.1 \mathrm{~mm} & \leq H \leq 1.1 \mathrm{~mm} \\
0^{\circ} & \leq \alpha \leq 90^{\circ}
\end{aligned}
$$


Choosing $P(\alpha)=\cos (\alpha)$ means that the pellet's orientation is 3D isotropic. Note that this is an assumption; there is no mechanism that would suggest a different distribution for the pellet orientation, because the pellets are rather fat cylinders, quite close to a sphere (a pencil shaped pellet, however, would align to the guiding tube direction). The second term $P(d, l, a \mid R, H, \alpha)$ on the RHS is called the likelihood; the likelihood is our knowledge about the experiment. In our case this term contains the PDF of the shadow parameters for given pellet parameters. This term can be evaluated independent from any measurement, based on the experimental setup and geometry. More details about the likelihood will be given later in this section.

The analysis continues by integrating the posterior PDF over the variable $\alpha$, since we are not interested in it:

$$
P(R, H \mid d, l, a)=\int_{\alpha} P(R, H, \alpha \mid d, l, a)
$$

In this way we get the 2-dimensional PDF of $R$ and $H$. This can be used to calculate the PDF of the pellet volume as follows:

$$
P(V(R, H) \mid d, l, a)=V(R, H) \cdot P(R, H \mid d, l, a)
$$

where $V(R, H)$ is the volume of a cylinder with radius $R$ and height $H$. Then the expected value of the pellet volume is calculated, which we regard as the output of the volume reconstruction algorithm:

$$
V_{\text {pel }}^{\text {reconstructed }}=E(V)=\iint_{R, H} V(R, H) \cdot P(V(R, H) \mid d, l, a)
$$

The uncertainty of the reconstructed volume is approximated by the standard deviation of the volume PDF: $\sigma_{V}{ }^{2}=E\left(V^{2}\right)-E^{2}(V)$.

Finally, we return to the likelihood $P(d, l, a \mid R, H, \alpha)$, the term that needs to be explained in eq. (3). It is the PDF of shadow parameters for a given pellet parameter range. It is calculated as follows:

$$
\begin{gathered}
P(d, l, a \mid R, H, \alpha) \sim \\
\sim \exp \left\{-\frac{(d-D(R, H, \alpha))^{2}}{2 \sigma_{D}{ }^{2}}\right\} \cdot \exp \left\{-\frac{(l-L(R, H, \alpha))^{2}}{2 \sigma_{L}{ }^{2}}\right\} \cdot \exp \left\{-\frac{(a-A(R, H, \alpha))^{2}}{2 \sigma_{A}{ }^{2}}\right\}
\end{gathered}
$$

where $D, L, A$ are the shadow parameters as functions of the pellet parameters. Two of these can be calculated analytically as already described by eq. (2). However, parameter $D$ can only be calculated numerically, therefore the likelihood must be calculated in a numerical simulation. For this, one has to abandon the continuous variables $R, H, \alpha$, and discrete variables $r_{i}, h_{j}, \alpha_{k}$ have to be used. This results only in a slight change in the volume reconstruction algorithm: integrals are substituted by summations. The number of discrete values for the variables $R, H, \alpha$ is denoted by $n_{r}, n_{h}, n_{\alpha}$, respectively (the number of bins taken in the possible ranges). The number of bins determines the computational need of the 
algorithm, as well as the accuracy of the result. This means that the number of elements in the likelihood PDF that must be calculated in order to evaluate a single measurement is $N=n_{r} \cdot n_{h} \cdot n_{\alpha}$. If the number of bins is sufficiently high, the result will be the same as it would be in the continuous case. The number of bins is optimal when the desired precision is reached with the smallest computational effort.

In eq. (5) $\sigma_{D}, \sigma_{L}$ and $\sigma_{A}$ are the measurement errors of the corresponding variables, determined by the image resolution and evaluation algorithm. The typical image resolution was found to be 23 pixels $/ \mathrm{mm}$; for $\sigma_{D}, \sigma_{L}$ the uncertainty is independent of the pellet size: two times 0.5 pixels (finding the two ends of a line), i.e. $0.043 \mathrm{~mm}$. For the uncertainty $\sigma_{A}$ one has to consider the circumference of the pellet shadow and multiply it by 0.5 pixels; the conservative upper boundary for this value (for the ideal Blower-gun pellet) is

$$
2 r_{\text {pel }} \pi \cdot 0.5 \text { pixel } \leq 2 \mathrm{~mm} \cdot \pi \cdot 0.0215 \mathrm{~mm} \sim 0.14 \mathrm{~mm}^{2}
$$

Since the pellet shadow can be completely separated from the background on the shadowgraphy image, $\sigma_{D}, \sigma_{L}$ and $\sigma_{A}$ do not depend on where the shadow is located on the image. The above estimated values can be regarded to be small in the sense that they cause relatively little error in the volume reconstruction algorithm, compared to the effect of using a single 2D image to determine the volume of a 3D object. Therefore, in the volume reconstruction algorithm $\sigma_{D}, \sigma_{\mathrm{L}}$ and $\sigma_{A}$ were chosen to have higher values $(0.1 \mathrm{~mm}, 0.1 \mathrm{~mm}$ and $0.2 \mathrm{~mm}^{2}$, respectively) - this allows to avoid numerical errors when using larger (and therefore fewer) bins, thereby the computational need can be considerably reduced.

\subsection{Testing the volume reconstruction method}

The pellet volume reconstruction method was tested to determine the error of the reconstruction that includes all effects described above. 200 randomly sized cylindrical pellets were simulated with their shadow produced onto simulated images with the same resolution as in the measurement. The radius and height of the simulated pellets ranged from $0.5 \mathrm{~mm}$ to $1.0 \mathrm{~mm}$, with any combination equally probable; this range of pellet sizes was chosen based on previous experience in studying the size of pellets produced by the Blower-gun. The relative difference between the original and reconstructed volume was calculated for each pellet. A scan in the number of bins in all three variables was carried out to check the sensitivity of the method and also the required computation time. It was found that the reconstruction accuracy saturates by increasing the number of bins, but the computation time increases radically. The optimum number of bins in $R$ and $H$ was found to be 40, and 30 in $\phi$, resulting in a computation time of about $260 \mathrm{~s}$ for 200 pellets. The statistics of the reconstruction algorithm with these settings can be seen on Figure 7. 


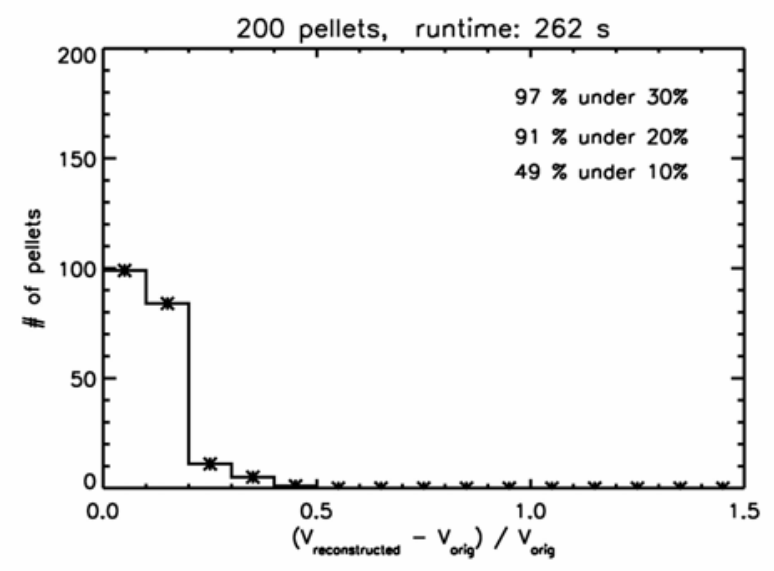

\section{Figure 7.: Distribution of relative difference of the pellet volume reconstruction algorithm.}

It can be seen that for most of the simulated cylindrical pellets (91\%) the reconstruction method provides a reconstructed mass within a $20 \%$ error. Therefore, average relative error of the volume reconstruction method will be considered $20 \%$.

\section{Results}

The shadowgraphy diagnostic and the Bayesian pellet volume reconstruction algorithm were applied to determine the pellet volume and delivery efficiency (reliability) of the Blower-gun in various setups. The evaluation is almost completely automatic, except for the threshold of the 1-bit conversion that has to be determined manually. First the variation of the pellet volume within a complete string was studied. Figure 8 shows the variations of the reconstructed pellet volume at both shadowgraphy locations within a single pellet string with repetition rate $f_{\text {pel }}=40 \mathrm{~Hz}$, under standard acceleration settings ( $p_{\text {prop }}=3.5 \mathrm{bar}$, $\tau_{\text {valve }}=2 \mathrm{~ms}$ ). The reconstructed volume varies between $1.5 \mathrm{~mm}^{3}$ and the maximum value for the $1^{\text {st }}$ location, with only a few exceptions, probably following the variation in the quality of the ice rod produced by the Blower-gun. Reconstructed volumes are mainly below $2 \mathrm{~mm}^{3}$ for the $2^{\text {nd }}$ location, showing mass degradation due to fragmentation and mass erosion due to the Leidenfrost-effect ${ }^{15}$. Note that the relative error of the reconstruction method is about $20 \%$.

Second, pellet survival in the guiding tube was studied. This can be best illustrated with pellet volume histograms before and after the tube. Figure 9 shows the statistics for the same pellet string as Figure 8. The (red) dotted line indicates the 'sound pellet' threshold, while the two black dashed lines correspond to the half and full pellet size. A 'sound pellet' was defined as a pellet with a reconstructed volume above $0.5 \mathrm{~mm}^{3}$ (са. 1/6 of the nominal size). The maximum number of sound pellets on a shadowgraphy image is one because always the largest shadow is evaluated only (see section 3). Note that the reconstruction algorithm can also produce reconstructed volumes larger than the ideal full size - this is the result of the $20 \%$ error of the reconstruction algorithm. The degradation of pellet mass due to the flight in the guiding tube is clearly visible: at the $1^{\text {st }}$ shadowgraphy location most pellets seem to be larger than half of the nominal size, while after the guiding tube about 2/3 of the pellets are below that level. 

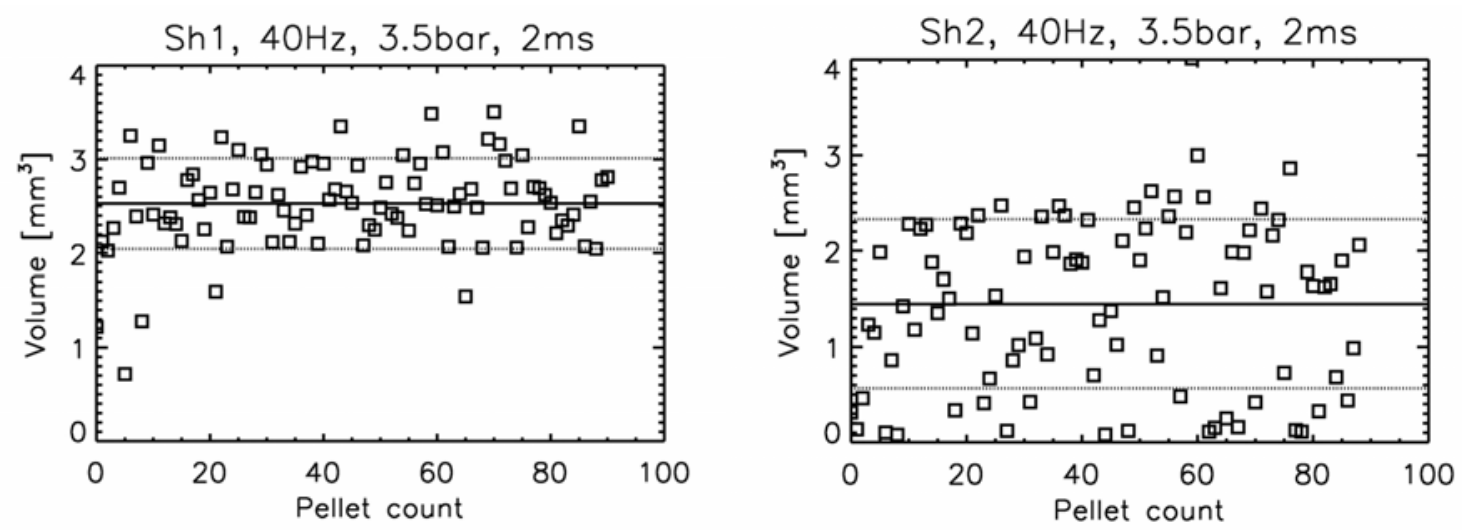

Figure 8.: Pellet volume variations within a string of pellets with injector settings $\mathbf{D}_{2}$ propellant gas @ 3.5 bar, 2 ms pulse length, $40 \mathrm{~Hz}$ injection frequency. Results from

before and after the guiding tube are presented on the left and right figures, respectively. Horizontal solid line: average volume, dotted lines: standard deviation from average.
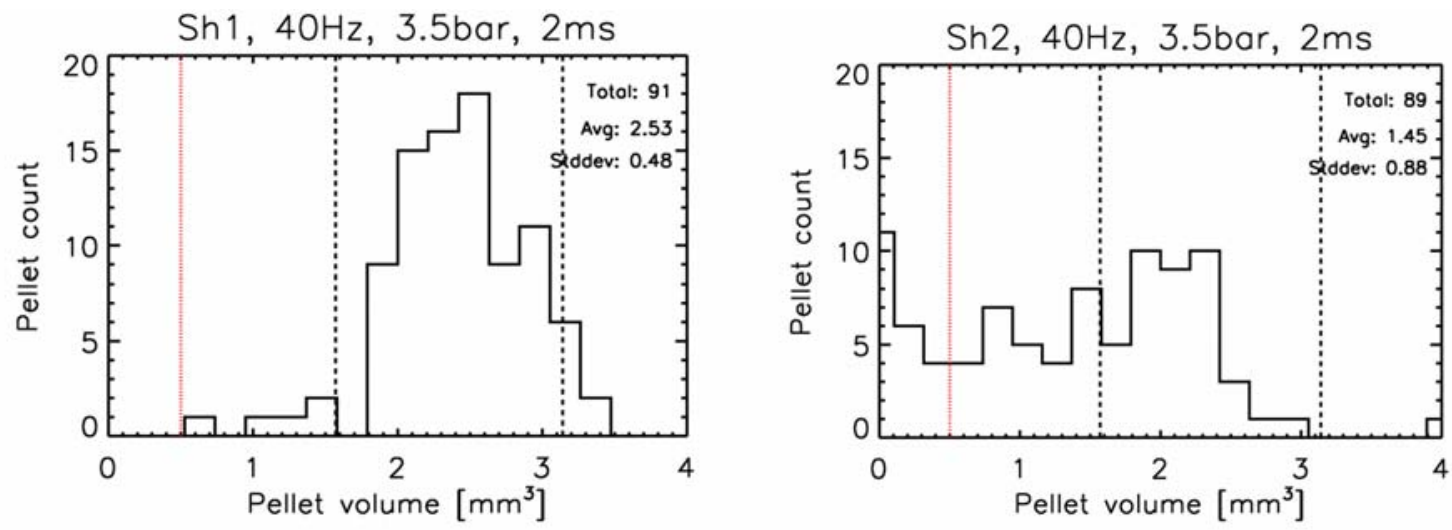

Figure 9.: Pellet volume histograms for $1^{\text {st }}$ and $2^{\text {nd }}$ shadowgraphy location (left and right, respectively). Dotted (red) line at $0.5 \mathrm{~mm}^{3}$ shows the preset threshold for 'sound' pellets. Dashed lines: half and full pellet size, respectively.

Another aspect of the offline testing was to study the pellet delivery efficiency. Efficiency was defined in two ways: absolute efficiency is the ratio of shadowgraphy images with a 'sound' pellet to the number of pellets requested (usually 110), whereas relative efficiency is the ratio of shadowgraphy images with a 'sound' pellet to the total number of shadowgraphy images with a detected pellet of any size:

$$
\eta_{\text {abs }}=\frac{N_{\text {sound_pellets }}^{\text {seen }}}{N_{\text {request }}}=\frac{N_{\text {sound_pellets }}^{\text {seen }}}{110}, \quad \eta_{\text {rel }}=\frac{N_{\text {sound_pellets }}^{\text {seen }}}{N_{\text {total }}^{\text {seen }}}
$$

Note that the absolute efficiency incorporates the efficiency of the shadowgraphy diagnostic: the camera system may miss pellets if they come too close after each other (e.g. a faster pellet can go close to a previous slower one, therefore the 'instantaneous' pellet frequency can exceed the maximum camera frame rate), or the light barrier may not detect some pellets etc. Note that the probability that this happens is quite low, because the camera frame rate is about $40 \mathrm{~Hz}$ higher than the maximum pellet rate. Therefore this effect may cause only a few \% change to the absolute efficiency. However, the $1^{\text {st }}$ camera had a maximum frame rate of 50 
$\mathrm{Hz}$ in these measurements, meaning that the absolute efficiency plot would contain the camera system triggering efficiency as well. Therefore, the efficiency values above $50 \mathrm{~Hz}$ for the $1^{\text {st }}$ camera were corrected by the ratio of the received and accepted triggers. This can be regarded as a good estimate of the real efficiency.

The efficiency tests of the Blower-gun consisted of two phases. In the first phase pellet delivery efficiency was studied for various pellet repetition rates (5-143 Hz) under standard acceleration settings. For frequencies higher than $100 \mathrm{~Hz}$ the gas pulse length was reduced to $0.7 \mathrm{~ms}$ in order to decrease the amount of propellant gas in the system. In the second phase the pellet frequency was held constant $(5,10$ or $40 \mathrm{~Hz})$ and the acceleration settings were varied ( $p_{\text {prop }}=3.5$ or 5 bar, $\tau_{\text {valve }}=2-10 \mathrm{~ms}$ ) in order to see the effect of these parameters on the velocity distribution of the pellets.

Figure 10 shows the results of the first test phase: It is clearly visible (right figure) that the reliability of the Blower-gun is almost constant for injection frequencies up to about $50 \mathrm{~Hz}$. Above this repetition rate the efficiency gradually decreases to about $55 \%$ for the $1^{\text {st }}$ diagnostic point, and even below $10 \%$ for the $2^{\text {nd }}$ camera, located after the guiding tube. This indicates that pellets produced at higher frequencies can more easily disintegrate in the guiding tube. These results seem to be in contradiction to data in ${ }^{9}$. However, most of the settings are different in the two analyses: in ${ }^{9}$ helium propellant gas was used with a pressure of 3 bar, and the test bed setup consisted of an almost straight pellet guiding tube - very much different from the setup in the present study, where deuterium propellant gas at a pressure of 3.5 bar was used and a guiding tube having two strong bends before the $2^{\text {nd }}$ diagnostic location (see Figure 1). All these could contribute to the differences in the results; however, the main difference comes from the evaluation algorithm: in ${ }^{9}$ pellets were detected by an acoustic impact detector at the end of the guiding tube, while in the present study the images of the $2^{\text {nd }}$ shadowgraphy location were evaluated for determining the final efficiency. The acoustic detector obviously cannot distinguish two pellet fragments arriving at the same time from the case when a completely sound pellet arrives to the detector - for the shadowgraphy this is just the opposite: even if the pellet is only broken into two parts, and both fragments are visible on the shadowgraphy image, the analysis only considers one of the fragments (the bigger one). The point in the latter evaluation process is that the penetration depth into the hot plasma is determined by the pellet (fragment) size, and not by the total amount of material. Therefore the determination of the pellet delivery efficiency in the present study is more conservative, and provides smaller reliability values.

On the left part of Figure 10 one can see that all pellets captured onto shadowgraphy images by the $1^{\text {st }}$ camera are exceeding the threshold of $0.5 \mathrm{~mm}^{3}$, but for the $2^{\text {nd }}$ camera this is not the case - another indication that pellets can be broken in the guiding tube, or their size can be reduced by at least a factor of 6 . Still, in almost all cases $80 \%$ of the pellets arriving at the end of the guiding tube are 'sound'. 

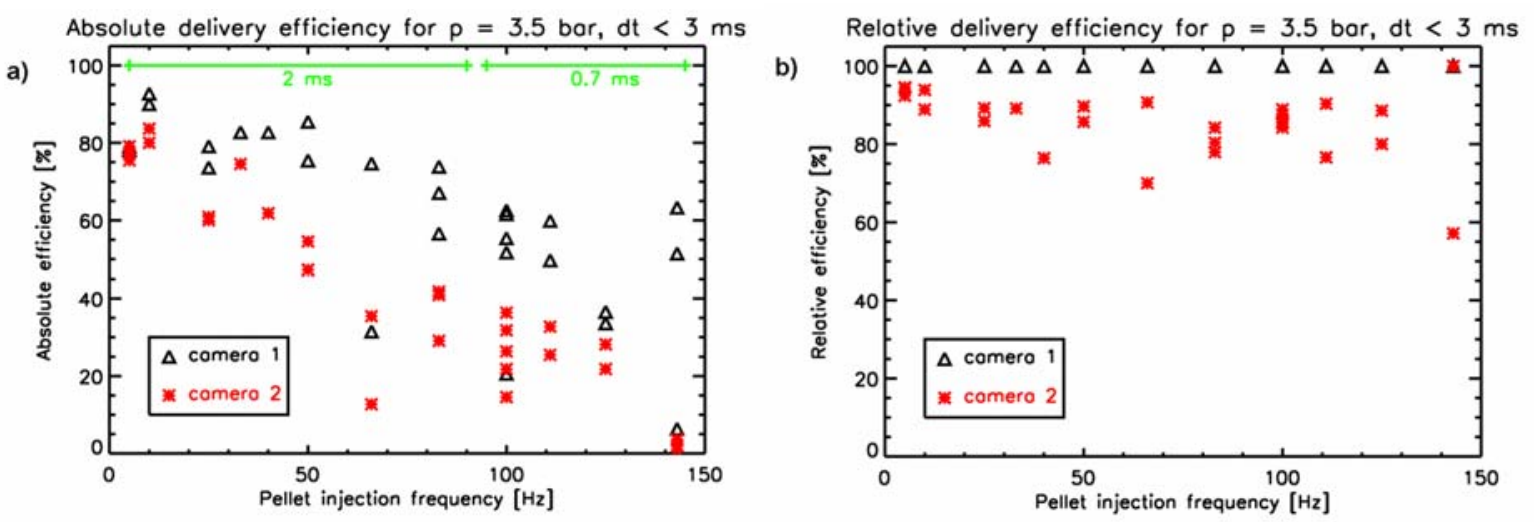

Figure 10.: Absolute (left) and relative (right) pellet delivery efficiency as function of pellet injection frequency. The gas pulse length for the different frequencies is indicated on the left figure. Note that the absolute efficiency plot includes the camera system triggering efficiency which decreases to about $30 \%$ at $143 \mathrm{~Hz}$ for camera 1.

On Figure 11 one can see the effect of the propellant gas pulse length $\left(\tau_{\text {valve }}\right)$. The top figures show that $\tau_{\text {valve }}$ does not really affect the efficiency for values less than 6 ms. Longer gas pulses have an adverse effect (especially at higher frequencies) since they cause additional heat conduction because of higher gas pressure in the system, which leads to the softening or melting/sublimation of the cryogenic pellet string. The excess gas can also hinder the acceleration, therefore pellets become slower and the velocity distribution broadens (see bottom left figure). The velocity of the pellets can mostly be affected by the propellant gas pressure, as can be seen on the bottom right figure: two short series of scans were completed for $p_{\text {prop }}=3.5$ and 5 bar with $\tau_{\text {valve }}=2,5$ and $7 \mathrm{~ms}$. Also on this figure it is clearly visible that the gas pulse length in the applied range has no real effect on the velocity distribution: the width and position of the distribution is very similar in all three cases for each pressure value. On the other hand, about 20\% higher velocities were measured for the 5 bar cases than in the 3.5 bar scenarios. Changing the propellant gas pressure to 5 bar did not have a considerable effect on the efficiency. 

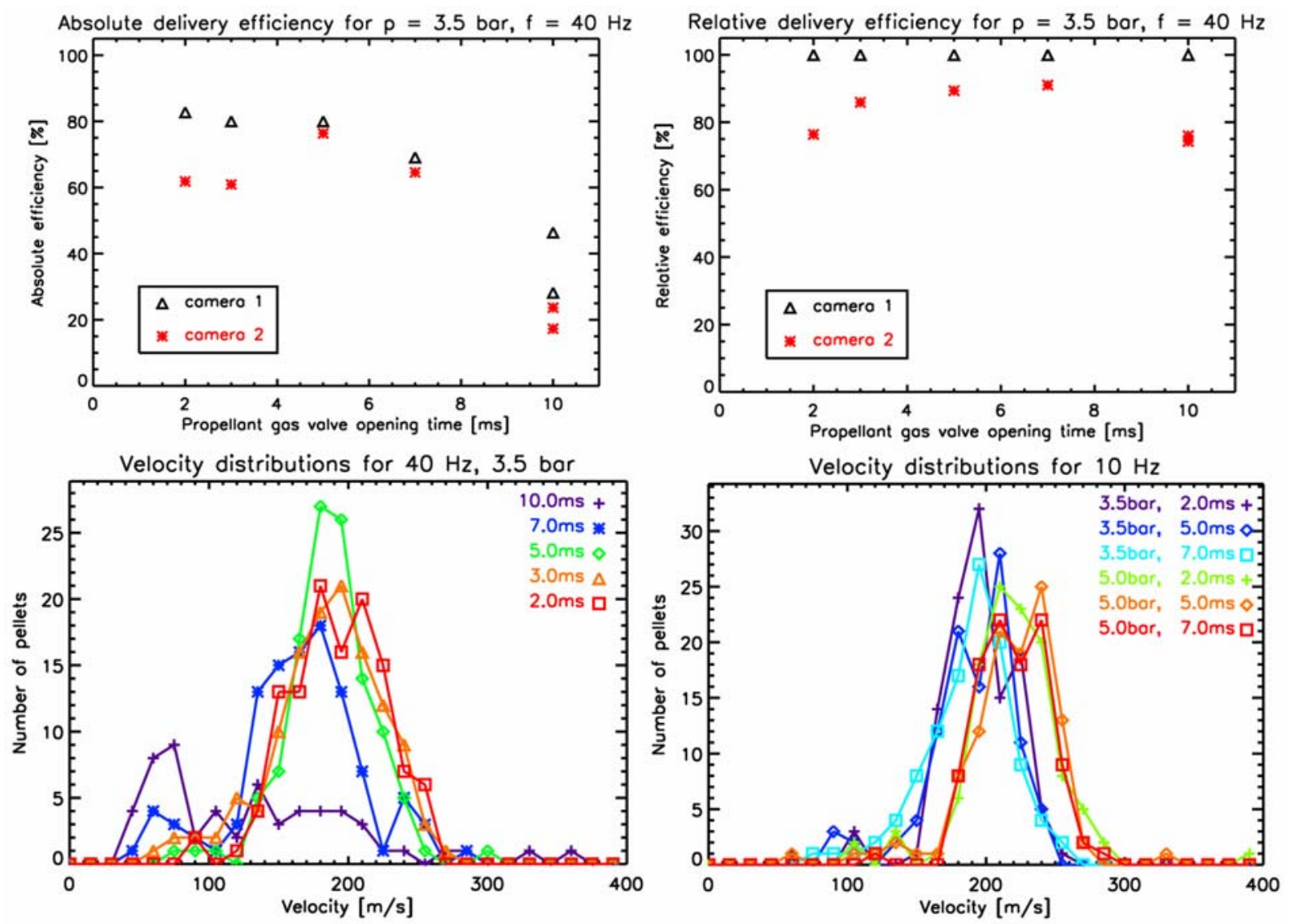

Figure 11.: Absolute (top left) and relative (top right) pellet delivery efficiency as a function of the propellant gas valve opening time (accelerating pulse length). The bottom left figure presents the velocity distributions for the same settings at the $2^{\text {nd }}$ shadowgraphy location, while the bottom right shows the same for six different setups.

\section{Conclusions}

A shadowgraphy system with two diagnostic locations was installed onto the new LFS pellet launcher (Blower-gun) at ASDEX Upgrade, allowing for the inspection of pellets just after the acceleration phase and after the guiding tube. The effect of the pellet injection frequency, propellant gas pressure and pulse length was studied in terms of pellet delivery efficiency and velocity distribution. Two different efficiencies were defined: the absolute efficiency also incorporates the diagnostics efficiency by relating the number of detected pellets to the number of requests, thereby providing a very conservative measure of the injector's reliability. The relative efficiency only considers pellets captured by the shadowgraphy system.

A Bayesian analysis was applied in order to reconstruct the volume of a detected pellet from a single 2D image of the pellet shadow produced by the shadowgraphy system. The analysis was based on the assumption that the observed shadows were produced by cylindrical pellets, i.e. the pellets preserve their shape throughout their flight in the guiding tube. During the evaluation a pellet was accepted to be 'sound' if the reconstructed volume was above 0.5 $\mathrm{mm}^{3}$, ca. $1 / 6$ of the initial pellet size. The main advantage of this analysis is that it is fully automatic, therefore it can be used for a quick evaluation of the measurement. 
In the first series of tests it was found that under standard propellant gas pressure and pulse length settings the absolute efficiency decreases considerably for pellet injection frequencies above $50 \mathrm{~Hz}$, while the relative efficiency is not really affected. The propellant gas pulse length has no effect on the efficiency or the velocity distribution, except for very large values ( $\tau_{\text {valve }}>6 \mathrm{~ms}$, three times the standard value). In these extreme cases gas blocking occurs: the pumping system cannot completely remove the propellant gas, which in turn hinders pellet acceleration and enhances heat conduction, causing the cryogenic pellets to soften or sublimate. The propellant gas pressure, on the other hand, has a mild effect on the velocity distribution: while the efficiency is almost not affected, higher pellet velocities can be measured for increased gas pressure scenarios, although the effect is not pronounced. Finally, the most reliable operating scenarios for the next campaigns were determined as follows: the standard propellant gas settings of $p_{\text {prop }}=3.5 \mathrm{bar}, \tau_{\text {valve }}=2 \mathrm{~ms}$ shall be used with the pellet injection frequency in the range of $f_{\mathrm{pel}}=5-50 \mathrm{~Hz}$.

\section{References}

${ }^{1}$ F. Wagner et al, Phys. Rev. Letters 49, 1408 (1982).

2 P. T. Lang et al, Nucl. Fusion 36, 1531 (1996).

${ }^{3}$ P. T. Lang and et al, Nucl. Fusion 43, 1110 (2003).

${ }^{4}$ L. R. Baylor et al, J. of Nucl. Materials 290-293, 398 (2001).

${ }^{5}$ H. Zohm, Plasma Phys. and Control. Fusion 38, 105 (1996).

${ }^{6}$ F. Federici et al, Plasma Phys. and Control. Fusion 45, 1523 (2003).

${ }^{7}$ P. T. Lang et al, Nucl. Fusion 45, 502 (2005).

${ }^{8}$ P. T. Lang et al, Nucl. Fusion 44, 665 (2004).

${ }^{9}$ P. T. Lang et al, Rev. Sci. Instrum. 78, 023504 (2007).

10 M. J. Gouge et al, Rev. Sci. Instrum. 61, 2102 (1990).

11 A. Lorenz et al, Fusion Eng. Des. 58-59, 325 (2001).

12 A. Lorenz et al, Fusion Eng. Des. 69, 15 (2003).

13 P. B. Parks et al, Phys. Fluids 21, 1735 (1978).

14 T. Bayes R. Price, Philos. Transactions (1683-1775) 53, 370 (1763).

15 P. T. Lang et al, Rev. Sci. Instrum. 74, 3974 (2003). 